Georgian Mathematical Journal

Volume 14 (2007), Number 2, 279-288

\title{
THE EXPONENTIAL STABILITY AND INSTABILITY OF DIFFERENTIAL SYSTEMS WITH RESPECT TO THE LINEAR COPPEL-CONTI APPROXIMATION. ESTIMATION OF CHARACTERISTIC EXPONENTS
}

\author{
NIKOLAI A. IZOBOV AND RIMMA A. PROKHOROVA \\ Dedicated to prominent mathematician I. Kiguradze, \\ Academician of the Georgian Acad. Sci., \\ on his 70th birthday anniversary
}

\begin{abstract}
We prove the exponential stability and instability of a trivial solution of perturbed nonlinear differential systems' with various CoppelConti linear approximations and perturbations of higher order smallness. We also obtain upper and lower estimates for characteristic exponents of systems of linear approximation and solutions of the considered perturbed systems.
\end{abstract}

2000 Mathematics Subject Classification: 34C41, 34D10.

Key words and phrases: Coppel-Conti linear systems, perturbation of higher order smallness, exponential stability and instability, Lyapunov characteristic exponents.

We consider the linear systems

$$
\dot{x}=A(t) x, \quad x \in R^{n}, \quad t \geq 0,
$$

with piecewise-continuous coefficients, unbounded, speaking, in general on the semi-axis $[0,+\infty)$ (and bounded on any segment $\left[0, t_{0}\right]$ ). Let $X_{A}(t), X_{A}(0)=E$ and $X_{A}(t, \tau)$ be respectively the fundamental matrix and the Cauchy matrix of system $\left(1_{A}\right)$. In the sequel, system $\left(1_{A}\right)$ will be identified with its matrix of coefficients $A$, while its belonging to some set $M$ will be written as the inclusion $A \in M$.

Definition 1 ([1, pp. 68, 74], [2], [3], [4, p. 40], [5]-[7]). The set of all linear systems $\left(1_{A}\right)$ for whose Cauchy matrices the condition

$$
C_{p}(A) \equiv \sup _{t \geq 0} \int_{0}^{t}\left\|X_{A}(t, \tau)\right\|^{p} d \tau<+\infty, \quad p>0,
$$

or the condition

$$
D_{p}(A) \equiv \sup _{t \geq 0} \int_{t}^{+\infty}\left\|X_{A}(t, \tau)\right\|^{p} d \tau<+\infty, \quad p>0,
$$

is fulfilled is called the Coppel-Conti sets $L^{p} S$ and $L^{p} N, p>0$, respectively. 
In the case of systems $\left(1_{A}\right)$ with integrally bounded coefficients on the semiaxis $[0,+\infty)$, these sets coincide $([6],[8])$ with $E S$ and $N S$, the sets of uniformly exponentially stable and unstable linear systems, respectively .

The sets $L^{p} D$ of linear systems $\left(1_{A}\right)$ are a generalization of the sets $L^{p} S$ and $L^{p} N, p>0$.

Definition 2 ([1, p. 131], [2]). We say that system $\left(1_{A}\right)$ belongs to the set $L^{p} D, p>0$, if for it there exists a pair of mutually complementary projectors $P_{1}$ and $P_{2}$ and a constant $K_{p}(A)$ such that the equality

$$
\int_{0}^{t}\left\|X_{A}(t) P_{1} X_{A}^{-1}(\tau)\right\|^{p} d \tau+\int_{t}^{+\infty}\left\|X_{A}(t) P_{2} X_{A}^{-1}(\tau)\right\|^{p} d \tau \leq K_{p}(A), \quad t \geq 0,
$$

is fulfilled.

It is obvious that if system $\left(1_{A}\right)$ belongs to the set $E D$ of exponentially dichotomous systems, then $A \in L^{p} D$ for any $p>0$. If however the matrix of coefficients $A$ of system $\left(1_{A}\right)$ is integrally bounded, then the inclusion $A \in L^{p} D$ with some $p>0$ implies [8] the inclusion $A \in E D$. In the general case, for any $p>0$ there holds [8] the representation

$$
E D=L^{p} D \cap L^{\infty} D
$$

where $L^{\infty} D$ denotes the set of linear systems with regular dichotomy on $R_{+}$.

The sets $L^{p} S$ and $L^{p} N$ are obviously the subsets of the set $L^{p} D$, corresponding respectively to the projectors $P_{1}=E$ and $P_{2}=0$ in the former case, and to the projectors $P_{1}=0$ and $P_{2}=E$ in the latter case. For the introduced sets we have $([6],[9],[10])$ the strict inclusions

$$
L^{p_{2}} S \subset L^{p_{1}} S, \quad L^{p_{2}} N \subset L^{p_{1}} N, \quad L^{p_{2}} D \subset L^{p_{1}} D, \quad p_{2}>p_{1}>0 .
$$

V. Coppel [1, pp. 68, 74] established the following properties of nontrivial solutions of system $\left(1_{A}\right)$ from the set $L^{1} D$ :

$$
\begin{gathered}
x(t) \rightarrow 0 \text { as } t \rightarrow+\infty \text { if } x(0) \in P_{1} R^{n} \\
\varlimsup_{t \rightarrow+\infty}\|x(t)\|=+\infty \text { if } x(0) \in P_{2} R^{n} .
\end{gathered}
$$

For any $p>0$, these properties of solutions of system $\left(1_{A}\right)$ from the set $L^{p} D$ are established similarly. Moreover, the first of properties (3) of solutions of system $\left(1_{A}\right)$ belonging to the set $L^{1} D$ is refined $[1$, p. 68] as follows:

$$
\|x(t)\| \leq C \exp \left[-K_{1}^{-1}(A) t\right], \quad x(0) \in P_{1} R^{n} .
$$

1. Estimates of the characteristic exponents of linear systems. From the preceding Coppel's estimate and inclusion (2) it follows that the characteristic exponents $\lambda[x] \equiv \varlimsup_{t \rightarrow+\infty} \frac{1}{t} \ln \|x(t)\|$ of nontrivial solutions $x(t)$ of system $\left(1_{A}\right)$, which belongs to the set $L^{p} S$ for some $p \geq 1$, are negative as these exponents are for solutions $x(t)$ of system $\left(1_{A}\right)$ from the set $L^{p} D$ for $p \geq 1$ with the initial conditions $x(0) \in P_{1} R^{n}$. 
There arises a problem of obtaining estimates of the characteristic exponents of nontrivial solutions of linear systems $\left(1_{A}\right)$ which belong to the sets $L^{p} S, L^{p} N$ and $L^{p} D$ for any fixed $p>0$.

Such estimates are obtained in [1] and the theorems formulated below.

Theorem 1. For any fixed value of the parameter $p \in(0,+\infty)$, any solution $x:[0,+\infty) \rightarrow R^{n} \backslash\{0\}$ of an $n$-dimensional system $\left(1_{A}\right)$ from the set $L^{p} S$ has the finite or infinite characteristic exponent $\lambda[x]$ that satisfies the estimate

$$
\lambda[x] \leq-\left[p C_{p}(A)\right]^{-1}<0, \quad p>0,
$$

with the constant $C_{p}(A)$ from the definition of the set $L^{p} S$.

Corollary 1. For the higher characteristic exponent $\lambda_{n}(A)$ of a system $A \in$ $L^{p} S, p>0$, we have the estimate $\lambda_{n}(A) \leq-\left[p C_{p}(A)\right]^{-1}<0$.

Corollary 2. A trivial solution of a system $A \in L^{p} S, p>0$, is exponentially stable.

In the case of systems $\left(1_{A}\right)$ from the set $L^{p} N, p>0$, we have established that the lower characteristic exponent $\lambda_{1}(A)$ is positive and its lower estimate is obtained.

Theorem 2. For the characteristic exponents $\lambda[x]$ of nontrivial solutions $x$ of a system $\left(1_{A}\right)$ that belongs to the set $L^{p} N$ with parameter $p>0$, the estimate

$$
\lambda[x] \geq\left[p D_{p}(A)\right]^{-1}>0
$$

is valid, where $D_{p}(A)$ is the constant from the definition of the set $L^{p} N$.

Corollary 3. For the lower characteristic exponent $\lambda_{1}(A)$ of a system $A \in$ $L^{p} N, p>0$, we have the estimate $\lambda_{1}(A) \geq\left[p D_{p}(A)\right]^{-1}>0$.

In the general case of the set $L^{p} D, p>0$, with zero mutually complementary projectors $P_{1}$ and $P_{2}, P_{1}+P_{2}=E$, we have [11] the following theorem which is analogous to Theorems 1 and 2 .

Theorem 3. Let an $n$-dimensional system $\left(1_{A}\right)$ with piecewise-continuous coefficients on the semi-axis $[0,+\infty)$ belong to the set $L^{p} D, p>0$, defined by the constants

$$
\begin{aligned}
& C_{p}(A) \equiv \sup _{t \geq 0} \int_{0}^{t}\left\|X_{A}(t) P_{1} X_{A}^{-1}(\tau)\right\|^{p} d \tau<+\infty \\
& D_{p}(A) \equiv \sup _{t \geq 0} \int_{t}^{+\infty}\left\|X_{A}(t) P_{2} X_{A}^{-1}(\tau)\right\|^{p} d \tau<+\infty .
\end{aligned}
$$

Then the characteristic exponents $\lambda\left[x_{1}\right]$ and $\lambda\left[x_{2}\right]$ of any of its solutions $x_{1}(t)$ and $x_{2}(t)$ with the initial vectors $x_{i}(0) \in P_{i} R^{n} \backslash\{0\}, i=1,2$, satisfy respectively estimates (4) and (5) with new constants (6). 
The upper and lower estimates - obtained in Theorems 1-3 and Corollaries 1 and 2 - of the characteristic exponents of solutions of systems $\left(1_{A}\right)$ from the considered sets $L^{p} S, L^{p} N$ and $L^{p} D, p>0$, admit a more precise definition provided that $\sup _{t \geq 0}\{\cdots\}$ in the definition of the constants $C_{p}(A)$ and $D_{p}(A)$ is replaced by the upper limit $\varlimsup_{t \rightarrow+\infty}\{\cdots\}$.

The assumption that the mutual inclination of subspaces of solutions $X_{A}(t) P_{1} R^{n}$ and $X_{A}(t) P_{2} R^{n}$ is separable from zero on $R_{+}$is not, generally speaking, fulfilled [10] for linear systems from the set $L^{p} D, p>0$, with nontrivial projectors $P_{1}$ and $P_{2}$, though such an assumption holds [12, Ch. IV, §3, p. 234] for exponentially dichotomic systems. Moreover, the angular convergence of the subspaces as $t \rightarrow+\infty$ may take place with any rate [11].

2. Asymptotic stability and instability of perturbed systems. We established in our earlier works [3], [6], [13], see also [14], that Coppel-Conti sets are open throughout the set of linear systems $\left(1_{A}\right)$ if and only if $p \geq 1$. Therefore, in particular, for any linear system $\left(1_{A}\right)$ belonging to one of the introduced Coppel-Conti sets with parameter $p \geq 1$ there exists $\varepsilon(A)>0$ such that the system $A+Q$ with any piecewise-continuous matrix $Q$, which satisfies the condition $\|Q(t)\|<\varepsilon(A), t \geq 0$, also belongs to the same set. Thus any linear system, sufficiently close to a system $A$ from the Coppel-Conti set with parameter $p \geq 1$, inherits all the properties of solutions of system $\left(1_{A}\right)$.

There arises the question whether the properties of asymptotic stability and, accordingly, instability of the system of Coppel-Conti linear approximation are inherited, and they are, then for what values of the parameter $p>0$ they are inherited by a trivial solution of the perturbed system

$$
\dot{y}=A(t) y+f(t, y), \quad y \in R^{n}, \quad t \geq 0,
$$

with any perturbation $f \in \cup_{m>1} F_{m}$ of higher order smallness, where $F_{m}$ is the set of vector functions $f:[0,+\infty) \times U_{\rho(f)} \rightarrow R^{n}, U_{\rho(f)} \equiv\left\{t \in R^{n}:\|y\|<\rho(f)\right\}$, piecewise-continuous with respect to $t \geq 0$ and continuous with respect to $y \in U_{\rho(f)}$ and satisfying the condition

$\|f(t, y)\| \leq L\|y\|^{m}, \quad(t, y) \in[0,+\infty) \times U_{\rho(f)}, \quad L=L(f)=$ const $>0, \quad m>1$, and called $m$-perturbations [15].

To provide a precise answer to this question in the case of linear approximations $\left(1_{A}\right)$ belonging to the set $L^{p} S$, we introduce

Definition 3. We denote by $L^{p} S_{1}, p>0$, the set of all linear systems $A \in$ $L^{p} S$, for which a trivial solution of system (7) with this linear approximation and any perturbation $f \in \cup_{m>1} F_{m}$ is asymptotically stable.

The construction of the set $L^{p} S_{1}$ of the considered linear systems $\left(1_{A}\right)$ is given [10] by

Theorem 4. The set $L^{p} S_{1} \subset L^{p} S$ of linear systems $\left(1_{A}\right)$ coincides with the set $L^{p} S$ if and only if the parameter $p$ belongs to the interval $[1,+\infty)$. 
The necessity of the conditions of this theorem is proved by using [16] the following lemma that yields a more general result.

Lemma 1. For any $p \in(0,1)$ and any $\sigma \leq 0$ and $m \geq 1$ there exist respectively a system $A \in L^{p} S$ and a vector function $f:[0,+\infty) \times R^{n} \rightarrow R^{n}$ (with bounded partial derivatives of any finite order on any segment $\left.\left[0, t_{0}\right]\right)$, piecewisecontinuous with respect to $t \geq 0$ and infinitely differentiable with respect to the variables $y_{1}, \ldots, y_{n}$, which satisfies the condition

$$
\|f(t, y)\| \leq L e^{\sigma t}\|y\|^{m}, \quad L=\text { const }>0, \quad(t, y) \in[0,+\infty) \times R^{n},
$$

and is such that a trivial solution of the perturbed system (7) is Lyapunov unstable.

Corollary of Theorem 4. A trivial solution of system (7) with a linear approximation $A \in L^{p} S, p \geq 1$, and any $m$-perturbation $f$ of order $m>1$ is asymptotically stable.

To get a full answer as to the influence of $m$-perturbations on the property of instability of a trivial solution of an unstable Coppel-Conti linear approximation from the set $L^{p} N$, we introduce [7]

Definition 4. Let us consider the set $L^{p} N_{1}, p>0$, of all linear systems $\left(1_{A}\right)$ from $L^{p} N$, for everyone of which and for any $m$-perturbation $f:[0,+\infty) \times$ $U_{\rho(f)} \rightarrow R^{n}, f \in F_{m}, m>1$, piecewise-continuous with respect to $t \in[0,+\infty)$ and continuous with respect to $y \in U_{\rho(f)}$, there exists a neighborhood of the origin of radius $\varepsilon(A, f)>0$ such that it takes a finite time for any nontrivial solution of the $m$-perturbed system (7), which at the initial moment of time $t=0$ belongs to the neighborhood $U_{\varepsilon(A, f)}$, to reach the boundary $\partial U_{\varepsilon(A, f)}$.

The description of this set which plays an important role in the investigation of the linear approximation of instability of differential systems with perturbations of higher order smallness is given [7] by

Theorem 5. The set $L^{p} N_{1} \subset L^{p} N$ of linear systems $\left(1_{A}\right)$ coincides with the set $L^{p} N$ if and only if the parameter $p$ belongs to the interval $[1,+\infty)$.

The proof of the necessity of the conditions of Theorem 5 follows from the next lemma which is of independent interest in the case $p \in(0,1)$.

Lemma $2([7])$. For any numbers $p \in(0,1)$ and $m \in(1,+\infty)$ there exist a two-dimensional system $A_{k}=A \in L^{p} N$ with piecewise-continuous coefficients on the semi-axis $[0,+\infty)$ and an m-perturbation $f_{p m}=f:[0,+\infty) \times R^{2} \rightarrow R^{2}$, piecewise-continuous with respect to $t$ and infinitely differentiable with respect to the variables $y_{1}$ and $y_{2}$ in the domain $(0,+\infty) \times R^{2}$ (with bounded partial derivatives of any finite order on the set $\left[0, t_{0}\right] \times R^{2}$ with any $\left.t_{0} \in(0,+\infty)\right)$, such that the two-dimensional system

$$
\dot{y}=A(t) y+f(t, y), \quad y \in R^{2}, \quad t \geq 0,
$$

has countable number of infinitely right-continuable solutions $y^{(n)}(t), n \in N$, which decrease - as $t \rightarrow+\infty$ - faster than any negative exponent (with Lyapunov 
characteristic exponents equal to $-\infty)$ and uniformly converge, on the semi-axis $[0,+\infty)$, to a trivial solution $y=0$ of this system $\left(y^{(n)}(t) \rightrightarrows 0, t \in[0,+\infty)\right)$.

Corollary of Lemma 2. The statement of Lemma 2 holds for n-dimensional initial linear systems $\left(1_{A}\right)$ belonging to the set $L^{p} N, p \in(0,1)$, and for perturbed systems (7) with $m$-perturbations $f, m>1$.

Corollary of Theorem 5. A trivial solution of system (7) with a linear approximation $A \in L^{p} N, p \geq 1$, and any $m$-perturbation $f$ of order $m>1$ is Lyapunov-unstable.

In the general case of a linear approximation $\left(1_{A}\right)$ from the set $L^{p} D$ with defining projectors $P_{1}$ and $P_{2}, P_{1}+P_{2}=E, P_{2} \neq 0$, the answer to the question as to the stability of a trivial solution with $m$-perturbation is given by

Theorem 6. If system $\left(1_{A}\right)$ belongs to the set $L^{p} D$ with a parameter $p \geq 1$ and the nontrivial second projector $P_{2}$ from Definition 2 , then a trivial solution of system (7) with any perturbation $f \in \cup_{m>1} F_{m}$ is unstable.

\section{Estimation of the characteristic exponent of solutions of perturbed} systems. The application of Theorems 1 and 3 and the principle of linear inclusion [17, p. 159] allow us to estimate the characteristic exponents of solutions of system (7).

Theorem 7. Let linear system $\left(1_{A}\right)$ belong to the set $L^{p} S$ for $p \geq 1$. Then the characteristic exponent $\lambda[y]$ of any solution $y$ with the initial condition $y(0)$ from a sufficiently small neighborhood $U_{\rho}$ of the origin of the perturbed system (7) with an $m$-perturbation $f$ of higher order smallness $(M>1)$ satisfies the estimate $\lambda[y] \leq-\left[\widetilde{C}_{1}(A)\right]^{-1}$, where

$$
\widetilde{C}_{1}(A)=\varlimsup_{t \rightarrow+\infty} \int_{0}^{t}\left\|X_{A}(t, \tau)\right\| d \tau .
$$

Proof. According to Theorem 4, any solution $y(t)$ tends to zero as $t \rightarrow+\infty$. Therefore, by the principle of linear inclusion, this solution is a solution of the linear system $\left(1_{A+Q_{y}}\right)$ with a piecewise-continuous matrix $Q_{y}(t) \rightarrow 0$ with respect to $t \geq 0$ as $t \rightarrow+\infty$. From Theorem 2 of [2] on the openness of the set $L^{p} S$ with respect to uniformly small perturbations it follows that the system $A+Q_{y}$ belongs to $L^{p} S$. The inclusions $A \in L^{1} S$ and $\left(A+Q_{y}\right) \in L^{1} S$ from the first property of (2) are also valid.

By virtue of the condition $\lim _{t \rightarrow+\infty}\left\|X_{A}(t)\right\|=0$ for system $A \in L^{1} S$ we obtain that the limit relation $\varlimsup_{t \rightarrow+\infty} \int_{t_{0}}^{t}\left\|X_{A}(t, \tau)\right\| d \tau=\widetilde{C}_{1}(A)$ is fulfilled for any $t_{0} \geq 0$. Now let $\varepsilon>0$ be so small that the inequality $2 \varepsilon\left(\widetilde{C}_{1}(A)+\varepsilon\right)^{2}<1$ is fulfilled. For this $\varepsilon$, we construct $t_{0}$ such that $\left\|Q_{y}(t)\right\|<\varepsilon^{2}$ for $t \geq t_{0}$. Then the definition of 
$\widetilde{C}_{1}(A)$ implies the existence of $t_{1} \geq t_{0}$ such that the estimate

$$
\int_{t_{0}}^{t}\left\|X_{A}(t, \tau)\right\| d \tau \leq \widetilde{C}_{1}(A)+\frac{\varepsilon}{2}
$$

holds for any $t \geq t_{1}$ and we therefore have the inequality

$$
J_{A}(t)=\int_{t_{1}}^{t}\left\|X_{A}(t, \tau)\right\| d \tau \leq C_{1}(A)+\frac{\varepsilon}{2}, \quad t \geq t_{1} .
$$

Let us show that the inequality

$$
J_{A+Q_{y}}(t) \equiv \int_{t_{1}}^{t}\left\|X_{A+Q_{y}}(t, \tau)\right\| d \tau<\widetilde{C}_{1}(A)+\varepsilon
$$

is fulfilled for every $t \geq t_{1}$. Assume the contrary that there exists $\theta \geq t_{1}$ such that the following conditions are fulfilled:

$$
J_{A+Q_{y}}(t)<\widetilde{C}_{1}(A)+\varepsilon \quad t \in\left[t_{1}, \theta\right], \quad J_{A+Q_{y}}(\theta)=\widetilde{C}_{1}(A)+\varepsilon .
$$

Using the Cauchy formula for the representation of the matrix $X_{A+Q_{y}}(t, \tau)$ and changing the order of integration, by virtue of the first assumption of the contrary statement we obtain the inequalities

$$
\begin{aligned}
J_{A+Q_{y}}(\theta) & \leq J_{A}(\theta)+\int_{t_{1}}^{\theta} d \tau \int_{\tau}^{\theta}\left\|X_{A}(\theta, \xi)\right\|\left\|Q_{y}(\xi)\right\|\left\|X_{A+Q_{y}}(\xi, \tau)\right\| d \xi \\
& \leq \widetilde{C}_{1}(A)+\frac{\varepsilon}{2}+\varepsilon^{2} \int_{t_{1}}^{\theta} d \xi \int_{t_{1}}^{\xi}\left\|X_{A}(\theta, \xi)\right\|\left\|X_{A+Q_{y}}(\xi, \tau)\right\| d \tau \\
& <\widetilde{C}_{1}(A)+\frac{\varepsilon}{2}+\varepsilon^{2}\left(\widetilde{C}_{1}(A)+\frac{\varepsilon}{2}\right)\left(\widetilde{C}_{1}(A)+\varepsilon\right)<\widetilde{C}_{1}(A)+\varepsilon,
\end{aligned}
$$

which contradict the second assumption of the contrary statement. By virtue of an arbitrary smallness of $\varepsilon$ the proven inequality implies that $\widetilde{C}_{1}\left(A+Q_{y}\right) \leq$ $\widetilde{C}_{1}(A)$, and thereby, by virtue of Theorem 1 and Remark 1 , the required estimate $\lambda[y]<-\left[\widetilde{C}_{1}(A)\right]^{-1}$ is valid for the characteristic exponent $\lambda[y]$ of the considered solution $y$ of the perturbed nonlinear system (7). This completes the proof of Theorem 7 .

Corollary of Theorems 4 and 7. A trivial solution of system (7) with a linear approximation $A \in L^{p} S, p \geq 1$, and any $m$-perturbation $f$ of order $m>1$ is exponentially stable.

When the system of linear approximation of $\left(1_{A}\right)$ belongs to the set $L^{p} D$ for $p \geq 1$, there arises the question about the existence of the corresponding manifold of solutions, vanishing at infinity, of the perturbed system (7) with 
perturbation $f \in U_{m>1} F_{m}$ of some higher order smallness $m_{f}=m>1$ and about the derivation of upper estimates of the characteristic exponents of these solutions. The following statement is valid.

Theorem 8. Let the system of linear approximation $\left(1_{A}\right)$ belong to the set $L^{p} D$ with a number $p \geq 1$ and the mutually complementary projectors $P_{1}$ and $P_{2}, P_{1}+P_{2}=E$, of ranks $k \in\{1, \ldots, n-1\}$ and $n-k$, respectively. Then for any $\delta \in(0,1)$ there exists such an $r$-neighborhood $U_{r} \subset U_{p}$ of the origin of radius $r>0$ defined by the conditions

$$
L\left[C_{1}(A)+D_{1}(A)\right](2 c)^{m} r^{m-1}<\delta, \quad r(c) \equiv 2 c r<\rho
$$

with a number $c \geq\left\|X(t) P_{1}\right\| \geq 1$ for $t \geq 0$ that

1) for any vector $y_{0} \in R^{n}$ with projection $P_{1 y_{0}} \in U_{r} \backslash\{0\}$ there exists, in the neighborhood $U_{p}$, a solution $u:[0,+\infty) \rightarrow U_{r(c)} \backslash\{0\}$ of the perturbed system $(7)$ with perturbation $f \in F_{m}$ of smallness order $m>1$ that satisfies the estimates

$$
\left\|u(0)-P_{1} y_{0}\right\| \leq \delta\left\|P_{1} y_{0}\right\|, \quad\|u(t)\| \leq(1+\delta) c\left\|P_{1} y_{0}\right\|, \quad t \geq 0
$$

and has a negative characteristic exponent $\lambda[u]$;

2) to any two different values $P_{1 y_{1}}, P_{2 y_{2}} \in U_{r} \backslash\{0\}$ there correspond at least two different (not coinciding on the entire semi-axis $[0,+\infty)$ ) solutions $u_{i}$ : $[0,+\infty) \rightarrow U_{r(c)} \backslash\{0\}, i=1,2$, of system (7). These solutions form a $k$ dimensional manifold $M_{k}$.

Proof. We begin by noting that according to [10], from the inclusion $A \in L^{p} D$, $p \neq 1$, with the mutually complementary projectors $P_{1}$ and $P_{2}$ we obtain an inclusion $A \in L^{1} D$ with the same projectors.

Applying the method of successive approximations

$$
\begin{aligned}
y_{k+1}(t)= & X(t) P_{1} y_{0}+\int_{0}^{t} X(t) P_{1} X^{-1}(\tau) f\left[\tau, y_{k}(\tau)\right] d \tau \\
- & \int_{t}^{+\infty} X(t) P_{2} X^{-1}(\tau) f\left[\tau, y_{k}(\tau)\right] d \tau, \\
& P_{1} y_{0} \in U_{r} \backslash\{0\}, \quad k \geq 0, \quad t \geq 0,
\end{aligned}
$$

we prove the existence, on the semi-axis $[0,+\infty)$, of a solution $y=u(t)$ of the $n$-dimensional system of integral equations

$$
\begin{aligned}
y(t)= & X(t) P_{1} y_{0}+\int_{0}^{t} X(t) P_{1} X^{-1}(\tau) f[\tau, y(\tau)] d \tau \\
& -\int_{t}^{+\infty} X(t) P_{2} X^{-1}(\tau) f[\tau, y(\tau)] d \tau, \quad t \geq 0 .
\end{aligned}
$$

To this end, on any segment $\left[0, t_{0}\right]$ of the time axis we define the equicontinuous sequence $\left\{y_{k}(t)\right\}$ of functions $y_{k}:[0,+\infty) \rightarrow U_{r(c)} \backslash\{0\}$ and, after that, obtain 
from it a subsequence uniformly converging, on this segment, to some continuous function $u(t)$. Thus we construct the continuous vector-function $u:[0,+\infty) \rightarrow$ $U_{r(c)} \backslash\{0\}$ and then establish that it is a nontrivial solution of the system of integral equations (10) and thereby a bounded and piecewise-differentiable solution, on the semi-axis $[0,+\infty)$, of the perturbed differential system (7). Applying Theorem 10 from [1, p. 74) to this system, we obtain the property $u(t) \rightarrow 0$ of this system as $t \rightarrow+\infty$. According to the principle of linear inclusion [17, p. 159] the vector-function $u(t)$ is a nontrivial solution of the linear system $\left(1_{A+Q_{u}}\right)$, too, with the matrix $Q_{u}(t) \rightarrow 0$ as $t \rightarrow+\infty$. Since the initial system $\left(1_{A}\right)$ belongs to the set $L^{1} D$, by Theorem 23 in [13] the system $\left(1_{A+Q_{u}}\right)$ also belongs to the set $L^{1} D$, but this time, generally speaking, with other mutually complementary projectors of the previous dimensions $k$ and $n-k$ and some constants $C_{1}\left(A+Q_{u}\right)>0$ and $D_{1}\left(A+Q_{u}\right)>0$ by means of which we define the belonging of the system $\left(1_{A+Q_{u}}\right)$ to $L^{1} D$. According to Theorem 3 , the characteristic exponent $\lambda[x]$ of any nontrivial solution $x$ of any system $\left(1_{A}\right)$ from the set $L^{1} D$ satisfies one of two mutually exclusive estimates

$$
\lambda[x] \leq-\left[C_{1}(A)\right]^{-1}<0, \quad \lambda[x] \geq\left[D_{1}(A)\right]^{-1}>0 .
$$

Therefore the characteristic exponent $\lambda[u]$ of the solution $u(t) \rightarrow 0$ as $t \rightarrow+\infty$ of a system $\left(1_{A+Q_{u}}\right) \in L^{1} D$ satisfies the required estimate $\lambda[u] \leq-\left[C_{1}(A+\right.$ $\left.\left.Q_{u}\right)\right]^{-1}<0$. This completes the proof of Theorem 8 .

\section{REFERENCES}

1. W. A. Coppel, Stability and asymptotic behavior of differential equations. D. C. Heath and Co., Boston, Mass., 1965.

2. R. Conti, On the boundedness of solutions of ordinary differential equations. Funkcial. Ekvac. 9(1966), 23-26.

3. R. Conti, Quelques propriétés de l'opérateur d'évolution. Colloq. Math. 18(1967), 73-75.

4. R. ConTI, Linear differential equations and control. Lecture notes of a course on control theory given at the University of Florence, February-May, 1975. Istituto Nazionale di Alta Mathematica (INDAM), Institutiones Mathematicae, Vol. I. Academic Press, LondonNew York, 1976.

5. R. Conti, On a class of asymptotically stable linear differential equations. Tôhoku Math. J. (2) 32(1980), No. 2, 279-282.

6. N. A. Izobov and R. A. Prokhorova, Unstable linear systems of R. Conti. (Russian) Vestnik Beloruss. Gos. Univ. Ser. I Fiz. Mat. Mekh. 1989, No. 2, 39-44, 79.

7. N. A. Izobov and R. A. Prokhorova, On solutions of a differential system with an unstable Coppel-Conti linear approximation. (Russian) Differ. Uravn. 41(2005), No. 1, 61-72; English transl.: Differ. Equ. 41(2005), No. 1, 61-73.

8. R. A. Prokhorova, On the interrelation between various forms of dichotomies. (Russian) Differ. Uravn. 37(2001), No. 8, 1125-1127; English transl.: Differ. Equ. 37. (2001), .Nno. 8, 1180-1183.

9. N. A. Izobov and R. A. Prokhorova, On a problem of R. Conti. (Russian) Differentsial'nye Uravneniya 23(1987), No. 5, 775-791.

10. R. A. Prokhorova, Linear systems with $L^{p}$-dichotomy. (Russian) Differentsial'nye Uravneniya 29(1993), No. 12, 2090-2096, 2205-2206 (1994); English transl.: Differential Equations 29(1993), No. 12, 1821-1828. 
11. N. A. Izobov and R. A. Prokhorova, On the characteristic Lyapunov and lower Perron exponents of solutions Coppel-Conti differential systems. (Russian) Differ. Uravn. 42(2006), No. 12, 1612-1625; English transl.: Differ. Equ. 42(2006), No. 12, 1682-1695.

12. Yu. L. Daletskil and M. G. Krein, Stability of solutions of differential equations in Banach space. (Russian) Nonlinear Analysis and its Applications Series. Nauka, Moscow, 1970; English transl.: Translations of Mathematical Monographs, Vol. 43. American Mathematical Society, Providence, R.I., 1974.

13. N. A. Izobov and R. A. Prokhorova, Coppel-Conti sets of linear systems. (Russian) Tr. Inst. Mat., Minsk 4(2000), 54-68.

14. N. A. Izobov and R. A. Prokhorova, Coppel-Conti sets of linear differential systems. Special issue dedicated to Victor A. Pliss on the occasion of his 70th birthday. J. Dynam. Differential Equations 15(2003), No. 2-3, 281-303.

15. R. E. Vinograd and N. A. Izobov, Solution of the Ljapunov stability problem by the first approximation. (Russian) Differencial'nye Uravneniya 6(1970), 230-242.

16. N. A. Izobov and R. A. Prokhorova, Asymptotic stability of a differential system with linear Coppel-Conti approximation. (Russian) Differ. Uravn. 40(2004), No. 12, 16081614, 1725; English transl.: Differ. Equ. 40(2004), No. 12, 1687-1693

17. B. F. Bylov, R. E. Vinograd, D. M. Grobman, and V. V. Nemytskiı̆, Theory of Ljapunov exponents and its application to problems of stability (Russian) Nauka, Moscow, 1966.

(Received 12.01.2007)

Authors' addresses:

N. A. Izobov

Institute of Mathematics of National Academy

of Sciences of Belarus

11, Surganova Str., Minsk, 220072

Belarus

E-mail: izobovn@mail.ru

R. A. Prokhorova

Belarussian State University

4, Nezavisimosti Ave., Minsk, 220030

Belarus 